\title{
QUANTITATIVE KOROVKIN THEOREMS FOR POSITIVE LINEAR OPERATORS ON $L_{p}$-SPACES
}

BY

\author{
H. BERENS AND R. DE VORE
}

\begin{abstract}
Let $\left(L_{n}\right)$ be a sequence of positive linear operators on $L_{p}(\Omega)$, $1<p<\infty$, or $C(\Omega)$ with $\Omega \subseteq R^{m}$. For suitable $\Omega$, the functions $\left(\varphi_{i}\right)_{i=0}^{m+1}$ given by $\varphi_{0}(x) \equiv 1, \varphi_{i}(x) \equiv x_{i}, 1<i<m$, and $\varphi_{m+1}(x) \equiv|x|^{2}$ form a test set for $L_{p}(\Omega)$. That is, if $L_{n}\left(\varphi_{i}\right)$ converges to $\varphi_{i}$ in $\|\cdot\|_{p}$ for each $i=0$, $1, \ldots, m+1$, then $L_{n}(f)$ converges to $f$ in $\|\cdot\|_{p}$ for each $f \in L_{p}(\Omega)$. We give here quantitative versions of this result. Namely, we estimate $\| f-$ $L_{n} f \|_{p}$ in terms of the error $\left\|\varphi_{i}-L_{n} \varphi_{i}\right\|_{p}, 0<i<m+1$, and the smoothness of the function $f$.
\end{abstract}

1. Introduction. Our interest in this paper is in quantitative Korovkin theorems for approximation by positive linear operators in $L_{p}$-spaces. Such theorems are well known for the space $C[a, b]$ of continuous functions on a compact interval. For example, let $\left(L_{n}\right)$ be a sequence of positive linear operators on $C[0,1]$ into itself, let $\varphi_{i}(x)=x^{i}, i=0,1,2$, and $\lambda_{n}=$ $\max _{i=0,1,2}\left\|L_{n} \varphi_{i}-\varphi_{i}\right\|_{\infty}$. Then for each $f \in C[0,1]$

$$
\left\|f-L_{n} f\right\|_{\infty} \leqslant C\left\{\lambda_{n}\|f\|_{\infty}+\omega_{2}\left(f, \sqrt{\lambda_{n}}\right)\right\}, \quad n=1,2, \ldots,
$$

with $\omega_{2}(\cdot, \delta), 0<\delta<1$, the 2 nd order modulus of smoothness as measured in $\|\cdot\|_{\infty}$ and $C$ an absolute constant (see G. Freud [6]).

An easy way to prove (1.1) is to establish the inequality

$$
\left\|f-L_{n} f\right\|_{\infty}<C \lambda_{n}\left\{\|f\|_{\infty}+\left\|f^{\prime \prime}\right\|_{\infty}\right\}
$$

for each $f \in C^{2}[0,1]$ and then use well-known results from the interpolation of linear operators (see e.g. [5]) to get (1.1).

The key to establishing $L_{p}$-estimates is to give an analogue of (1.2) for $L_{p}$-approximation. This is done in $\$ 2$ for the spaces $X_{p}=L_{p}\left(I^{m}\right), 1<p<$ $\infty$, and $X_{p}=C\left[I^{m}\right]$ when $p=\infty$, with $I^{m}=[0,1]^{m}$ the unit $m$ cube.

If $r \geqslant 0$ and $1 \leqslant p \leqslant \infty$, we let $W_{p}^{r}\left(I^{m}\right)$ be the Sobolev space (see e.g. [1]) of all functions $f \in L_{p}\left(I^{m}\right)$ such that $D^{\alpha} f$ exists (in the Sobolev sense) and is

Received by the editors November 19, 1976 and, in revised form, June 22, 1977.

AMS (MOS) subject classifications (1970). Primary 41A35; Secondary 46E30, 46EB5, 41A25.

Key words and phrases. Positive linear operators, quantitative estimates, degree of approximation, Korovkin theorems.

1 This author was supported in part by the National Science Foundation Grant MCS 7605847 and the Alexander von Humbold Stiftung. 
in $L_{p}$ for all $\alpha \in Z_{0}^{m},|\alpha| \leqslant m$. The norm on $W_{p}^{r}\left(I^{m}\right)$ is

$$
\|f\|_{r, p}=\max _{|\alpha|<r}\left\|D^{\alpha} f\right\|_{p}
$$

with of course the $L_{p}$-norm taken over $I^{m}$.

The set of functions $\left\{\varphi_{0}, \ldots, \varphi_{m+1}\right\}$ with for each $x \in I^{m} \varphi_{0}(x) \equiv 1$, $\varphi_{i}(x)=x_{i}, 1 \leqslant i \leqslant m, \varphi_{m+1}(x)=|x|^{2}$ forms a test set for $X_{p}, 1<p \leqslant \infty$. If $L$ is a positive linear operator mapping $X_{p}$ into itself, let

$$
\lambda_{p}=\max _{0<i<m+1}\left\|L \varphi_{i}-\varphi_{i}\right\|_{p} .
$$

In Theorem 1, we show that for each $f \in W_{\infty}^{2}$

$$
\|f-L f\|_{p}<C\|f\|_{2, \infty} \lambda_{p},
$$

with $C$ a constant depending only on the dimension $m$. Of course, when $\left(L_{n}\right)$ is a sequence of operators then (1.4) gives an $L_{p}$-analogue of (1.2).

This result may seem a little uncomfortable since the right-hand side involves the $W_{\infty}^{2}$-norm which does not depend on $p$. However this estimate is sharp in a very strong sense. Namely, we also show in $\$ 2$ that for each $f$ with continuous 2 nd partials we have

$$
\sup _{\substack{L>0 \\ L \neq I}} \frac{\|f-L f\|_{p}}{\lambda_{p}} \geqslant C\|f\|_{2, \infty},
$$

with $C$ an absolute constant.

In order to extend (1.4) to give estimates in terms of $L_{p}$-smoothness, we seek smoothness spaces that can be embedded in $W_{\infty}^{2}$. This is discussed in $\S 3$ where we establish certain known embedding theorems for Besov spaces. Using interpolation theory, these embeddings give quantitative estimates for approximation of functions in other $L_{p}$-smoothness spaces, for example the classical Lipschitz spaces.

As we have pointed out, all our results rest on (1.4). Thus the quantitative theory for approximation by positive linear operators sits naturally in $L_{\infty}$. This should be compared with the quantitative estimates for positive contractions. Here for $L_{p}(I), 1 \leqslant p<\infty$, we have shown in [2] that if $L$ is a positive linear contraction on $L_{p}(I)$ and $\eta_{p}=\max _{i=0,1}\left\|\varphi_{i}-L \varphi_{i}\right\|_{p}$ then for any $f \in L_{p}(I)$

$$
\|f-L f\|_{p} \leqslant C\|f\|_{2,1} \eta_{p}^{1 / p}
$$

with $C$ a constant. The remainder of the quantitative theory for $L_{p}(I)$ was built up from (1.5). Thus the theory for contractions is essentially an $L_{1^{-}}$theory.

The form of the present paper is to prove everything completely for $L_{p}\left(I^{m}\right)$. All the theorems have extensions to more general regions and we point them out in $\$ 4$. 
2. The fundamental estimate. In this and the next section, we work only on $I^{m}$ and so we do not indicate the dependence of the spaces on the domain but write instead $L_{p}$ for $L_{p}\left(I^{m}\right), W_{p}^{r}$, etc. Let $\lambda_{p}$ be as defined in (1.3). One main result is the following

TheOREM 1 . Let $1 \leqslant p \leqslant \infty$ and $L$ be any positive linear operator mapping $X_{p}$ into itself. If $f \in W_{\infty}^{2}$, then

$$
\|f-L f\|_{p} \leqslant C\|f\|_{2, \infty} \lambda_{p}
$$

with $C$ a constant depending only on the dimension $m$.

Proof. We prove only the case $1<p<\infty$ which is more complicated than the case $p=\infty$. We start with any $\varepsilon=k^{-1}, k$ a positive integer. We will estimate on $\mathcal{T}=\operatorname{int}\left(I^{m}\right)$. Using a uniform grid, we can decompose $\mathcal{T}$ into a union of $N=(m k)^{m}$ cubes, $\mathcal{T}=\cup_{i}^{N} Q_{i}$, where the $Q_{i}$ 's are all congruent to the cube $Q=\left\{x: 0<x_{i}<m^{-1} \varepsilon\right\}$ and are pairwise disjoint.

When $1 \leqslant i \leqslant N$, let $\xi^{(i)}$ be the center of $Q_{i}$ and define

$$
l_{i}(x)=f\left(\xi^{(i)}\right)+\left\langle\nabla f\left(\xi^{(i)}\right), x-\xi^{(i)}\right\rangle
$$

with $\nabla f$ the gradient of $f$ and $\langle\cdot, \cdot\rangle$ denoting the scalar product. Thus $l_{i}$ is the linear part of the Taylor expansion of $f$ at $\xi^{(i)}$. A simple estimate shows that for any $i$ and any $x$

$$
\begin{aligned}
\left|f(x)-l_{i}(x)\right| & <\mid \int_{0}^{1}(1-t)\left\{\sum_{1<\mu, \nu<m} \frac{\partial^{2} f}{\partial x_{\mu} \partial x_{\nu}}\left(\xi^{(i)}+t\left(x-\xi^{(i)}\right)\right)\right. \\
& \left.\left.\leqslant \frac{1}{2}\|f\|_{2, \infty} \sum_{1<\mu, \nu<m} \mid x_{\mu}-\xi_{\mu}^{(i)}\right)\left(x_{\nu}-\xi_{\nu}^{(i)}\right)\right\} d t \mid \\
& \leqslant \frac{m}{2}\|f\|_{2, \infty}\left|x-\xi_{\nu}^{(i)}\right|^{2} .
\end{aligned}
$$

To estimate $L f-f$, we use the fact that for each $i=1, \ldots, N$ and almost all $x \in Q_{i}$

$$
L(f, x)-f(x)=L\left(f-l_{i}, x\right)+L\left(l_{i}, x\right)-l_{i}(x)+l_{i}(x)-f(x),
$$

and consequently for almost all $x \in I^{m}$

$$
\begin{aligned}
|L(f, x)-f(x)| \leqslant & \sum_{i}\left|L\left(f-l_{i}, x\right)\right| \chi_{Q_{i}}(x) \\
& +\sum_{i}\left|L\left(l_{i}, x\right)-l_{i}(x)\right| \chi_{Q_{i}}(x)+\sum_{i}\left|l_{i}(x)-f(x)\right| \chi_{Q_{i}}(x) \\
= & S_{1}(x)+S_{2}(x)+S_{3}(x) .
\end{aligned}
$$


First, we want to estimate the norm of $S_{1}$. Because of (2.3),

$$
\left|L\left(f-l_{i}, x\right)\right|<\frac{m}{2}\|f\|_{2, \infty} L\left(\left|\cdot-\xi^{(i)}\right|^{2}, x\right) \text { a.e. }
$$

Recall the functions $\varphi_{v}, \nu=0,1, \ldots, m+1$, introduced in $\$ 1$. Then

$$
\begin{aligned}
& L\left(\left|\cdot-\xi^{(i)}\right|^{2}, x\right) \\
&= L\left(\varphi_{m+1}, x\right)-\varphi_{m+1}(x)-2 \sum_{\nu=1}^{m} \varphi_{\nu}\left(\xi^{(i)}\right)\left\{L\left(\varphi_{v}, x\right)-\varphi_{\nu}(x)\right\} \\
&+\varphi_{m+1}\left(\xi^{(i)}\right)\left\{L\left(\varphi_{0}, x\right)-\varphi_{0}(x)\right\}+\left|x-\xi^{(i)}\right|^{2} \text { a.e. } \\
&<\left|L\left(\varphi_{m+1}, x\right)-\varphi_{m+1}(x)\right|+2 \sum_{\nu=1}^{m}\left|L\left(\varphi_{\nu}, x\right)-\varphi_{\nu}(x)\right| \\
&+\left|L\left(\varphi_{0}, x\right)-\varphi_{o}(x)\right|+\left|x-\xi^{(i)}\right|^{2} \text { a.e. }
\end{aligned}
$$

giving

$$
\begin{aligned}
& S_{1}(x)<\frac{m}{2}\|f\|_{2, \infty}\left(\left|L\left(\varphi_{m+1}, x\right)-\varphi_{m+1}(x)\right|\right. \\
& \quad+2 \sum_{\nu=1}^{m}\left|L\left(\varphi_{\nu}, x\right)-\varphi_{\nu}(x)\right| \\
& \left.\quad+\left|L\left(\varphi_{0}, x\right)-\varphi_{0}(x)\right|+\frac{\varepsilon^{2}}{4 m}\right)
\end{aligned}
$$

and consequently

$$
\left\|S_{1}\right\|_{p}<\frac{m}{2}\|f\|_{2, \infty}\left(2(m+1) \lambda_{p}+\varepsilon^{2}\right)
$$

which is our estimate for $S_{1}$.

Now we want to estimate the norm of $S_{2}$. In this case, for each $i=1$, $2, \ldots, N$ and almost all $x \in Q_{i}$

$$
\begin{aligned}
\left|L\left(l_{i}, x\right)-l_{i}(x)\right|< & \|f\|_{\infty}\left|L\left(\varphi_{0}, x\right)-\varphi_{0}(x)\right| \\
& +\sum_{\nu=1}^{m}\left\|\frac{\partial f}{\partial x}\right\|_{\infty}\left|L\left(\varphi_{\nu}, x\right)-\varphi_{\nu}(x)\right| \\
& +\sum_{\nu=1}^{m}\left\|\frac{\partial f}{\partial x}\right\|_{\infty}\left|L\left(\varphi_{0}, x\right)-\varphi_{0}(x)\right| \text { a.e. }
\end{aligned}
$$

Hence

$$
S_{2}(x)<\|f\|_{1, \infty}\left\{\left|L\left(\varphi_{0}, x\right)-\varphi_{0}(x)\right|+2 \sum_{\nu=1}^{m}\left|L\left(\varphi_{\nu}, x\right)-\varphi_{\nu}(x)\right|\right\}
$$

giving

$$
\left\|S_{2}\right\|_{p}<(2 m+1)\|f\|_{1, p} \lambda_{p}
$$


Using the estimate (2.3) we obtain for $S_{3}$

$$
\left\|S_{3}\right\|_{p}<\|f\|_{2, m} \varepsilon^{2} \text {. }
$$

If we combine the estimates (2.6), (2.7), and (2.8) and use the fact that $\varepsilon$ can be chosen arbitrarily small, we get

$$
\|L f-f\|_{p}<(m+1)(m+2) \lambda_{p}\|f\|_{2, \infty},
$$

which completes the proof of the theorem.

We now want to give some examples which will show that the estimate (2.1) of Theorem 1 is sharp. For $f \in W_{\infty}^{2}$ and $1<p<\infty$, define

$$
\alpha_{p}(f)=\sup _{\substack{L>0 \\ L \neq I}} \frac{\|f-L f\|_{p}}{\lambda_{p}}
$$

The estimate (2.1) is equivalent to

$$
\alpha_{p}(f)<C\|f\|_{2, \infty} .
$$

We will now show that the reverse inequality also holds.

Let $1 \leqslant p<\infty$ (for $p=\infty$ one makes suitable modifications in our argument), $\eta \in R^{m}$ with $|\eta|=1$, and $x_{0} \in \operatorname{Int}\left(I^{m}\right)$. For $t>0$ small enough, the cube $S_{t}$ with center $x_{0}$ and sides of length $t$ is contained in $I^{m}$. We work from here on only with values of $t$ where $S_{2 t} \subseteq I^{m}$. For such $t$, define

$$
L_{t}(f, x)= \begin{cases}f(x), & x \notin S_{t}, \\ \frac{1}{2 t} \int_{-t}^{t} f(x+u \eta) d u, & x \in S_{t} .\end{cases}
$$

Then, $L_{t} \varphi_{i}=\varphi_{i}, 0 \leqslant i \leqslant m$, and

$$
\lambda_{t p}=\left\|L_{t} \varphi_{m+1}-\varphi_{m+1}\right\|_{p}=\frac{t^{2}}{3}\left\|\chi_{s_{t}}\right\|_{p}=\frac{1}{3} t^{2+m / p} .
$$

If $f \in W_{\infty}^{3}$, we let $d_{\eta}(f, x)=\langle\nabla f(x), \eta\rangle$ be the partial derivative of $f$ in the direction of $\eta$ at $x$ and $d_{\eta}^{2}(f, x)=d_{\eta}\left(d_{\eta}(f), x\right)$.

For $x \in S_{t}$ and $0<u<t$, Taylor's formula gives

$$
f(x+u \eta)+f(x-u \eta)-2 f(x)=u^{2} d_{\eta}^{2}(f, x)+\theta\left(u^{3}\right)
$$

with the $\theta$ independent of $x$ and $u$. Hence, returning to the definition of $L_{t}$, we have

$$
\left\|L_{2} f-f\right\|_{p}=\left(\frac{1}{4 t} \int_{-t}^{t} u^{2} d u\right)\left\|d_{\eta}^{2}(f, x) \chi_{s_{t}}(x)\right\|_{p}+\theta\left(t^{3}\left\|\chi_{s_{t}}\right\|_{p}\right) .
$$

Dividing by $\lambda_{t, p}$ and taking the limit as $t \rightarrow 0+$, gives

$$
\alpha_{p}(f) \geqslant \varlimsup_{t \rightarrow 0} \frac{\left\|L_{t} f-f\right\|_{p}}{\lambda_{t, p}}=\frac{1}{2} d_{\eta}^{2}\left(f, x_{0}\right),
$$


where we used the fact that $d_{\eta}^{2}(f, x)$ is a continuous function.

What we have just shown above is that for any $f \in W_{\infty}^{3}$ and any $\eta$ with $|\eta|=1$, we have $\left\|d_{\eta}^{2}(f)\right\|_{\infty} \leqslant 2 \alpha_{p}(f)$. Thus, for example, if we take $\eta=$ $\left(\delta_{i v}\right)_{v=0}^{m}$ (Kronecker delta notation) then we find

$$
\left\|\frac{\partial^{2} f}{\partial x_{i}^{2}}\right\|_{\infty} \leqslant 2 \alpha_{p}(f), \quad i=1,2, \ldots, m .
$$

We can also get an estimate for the mixed partials by taking $\eta=2^{-1 / 2}\left(\delta_{i p}+\right.$ $\left.\delta_{j v}\right)_{\nu=0}^{m}$ to find

$$
\left\|\frac{\partial^{2} f}{\partial x_{i}^{2}}+2 \frac{\partial^{2} f}{\partial x_{i} \partial x_{j}}+\frac{\partial^{2} f}{\partial x_{j}^{2}}\right\|_{p} \leqslant 4 \alpha_{p}(f)
$$

and so

$$
2\left\|\frac{\partial^{2} f}{\partial x_{i} \partial x_{j}}\right\|_{\infty} \leqslant 4 \alpha_{p}(f)+\left\|\frac{\partial^{2} f}{\partial x_{i}^{2}}\right\|_{\infty}+\left\|\frac{\partial^{2} f}{\partial x_{j}^{2}}\right\|_{\infty}<8 \alpha_{p}(f) .
$$

This shows that all second order partial derivatives can be estimated by $\alpha_{p}(f)$.

We can also estimate the first order partial derivatives of $f$. Now with the same notation as above, we define the operators

$$
L_{t}(f, x)= \begin{cases}f(x), & x \notin S_{t}, \\ \frac{1}{t} \int_{0}^{t} f(x+\eta u) d u, & x \in S_{t} .\end{cases}
$$

For these operators $L_{t}$, we have only $\varphi_{0}$ preserved. However, a simple calculation shows that $\left\|L_{t}\left(\varphi_{i}\right)-\varphi_{i}\right\|_{p}=\frac{1}{2}\left|\eta_{i}\right| t^{1+m / p}, i=1,2, \ldots, m$, and $\left\|L_{t}\left(\varphi_{m+1}\right)-\varphi_{m+1}\right\|_{p} \leqslant 2 t^{1+m / p}$. Hence, arguing similar to what we did above for the second partial derivatives we find

$$
\left|d_{\eta}\left(f, x_{0}\right)\right| \leqslant C \varlimsup_{t \rightarrow 0} \frac{\left\|L_{t} f-f\right\|_{p}}{\lambda_{t, p}} \leqslant C \alpha_{p}(f)
$$

with $C$ a constant depending only on $m$. From this it follows that $\left\|\partial f / \partial x_{i}\right\|_{\infty}$ $\leqslant C \alpha_{p}(f), i=1,2, \ldots, m$.

Hence, the norms of the first partial derivatives of $f$ can be estimated in terms of $\alpha_{p}(f)$.

The same ideas can be used to estimate the norm of $f$ itself. Now, take $L_{t}$ as

$$
L_{t}(f, x)= \begin{cases}f(x), & x \notin S_{t}, \\ (1+t) f(x), & x \in S_{t} .\end{cases}
$$

Then we have 


$$
\left|f\left(x_{0}\right)\right| \leqslant C \varlimsup_{t \rightarrow 0} \frac{\left\|L_{t} f-f\right\|_{p}}{\lambda_{t, p}} \leqslant C \alpha_{p}(f)
$$

with $C$ depending only on $m$. Thus, $\|f\|_{\infty} \leqslant C \alpha_{p}(f)$. Combining all the estimates above we get that for each $f \in W_{\infty}^{3}$,

$$
\|f\|_{2, \infty} \leqslant C \alpha_{p}(f)
$$

which is the converse to (2.9). By a denseness argument this inequality holds for all $f$ in $C^{2}$. This shows that in a very strong sense we cannot improve the estimate (2.1) save for the constant $C$.

3. Estimates in terms of $L_{p}$-smoothness. In this section, we want to obtain quantitative estimates in terms of $L_{p}$-smoothness. We continue to work on the cube $I^{m}$. If $\theta>0$, let $r$ be the smallest integer strictly larger than $\theta$ and let $1 \leqslant q \leqslant \infty$. The Besov spaces $B_{p}^{\theta, q}=B_{p}^{\theta, q}\left(I^{m}\right)$ are defined as the set of all functions $f \in L_{p}$ such that the norm

$$
\|f\|_{p}^{\theta, q}= \begin{cases}\|f\|_{p}+\left\{\int_{0}^{1}\left(t^{-\theta} \omega_{r, p}(f, t)\right)^{q} \frac{d t}{t}\right\}^{1 / q}, & 1 \leqslant q<\infty, \\ \|f\|_{p}+\sup _{0<t<1} t^{-\theta} \omega_{r}(f, t), & q=\infty,\end{cases}
$$

is finite. Here, $\omega_{r, p}(\cdot, \delta), 0<\delta \leqslant 1$, is the $r$ th order modulus of smoothness measured in $L_{p}$. The case $q=\infty$ gives the classical Lipschitz spaces $\operatorname{Lip}\left(\theta, r ; L_{p}\right)$. The parameter $\theta$ is the primary scaling while $q$ gives a finer scaling when $p$ and $\theta$ are held fixed.

For $0<\theta<r, r$ an integer, the spaces $B_{p}^{\theta, q}$ are precisely the interpolation spaces generated by the Peetre $\mathcal{H}$-method of interpolation between $L_{p}$ and $W_{p}^{r}$, see e.g. [4]. This follows from the fact that the modulus of smoothness and the $K$-functional, defined by

$$
\left.K_{r p}(f, t)=\inf \left\{\|f-g\|_{p}+t\|g\|_{r p}\right\}: g \in W_{p}^{r}\right\}, \quad t>0,
$$

are related by

$$
\begin{aligned}
C^{-1}\left\{\min \left(1, t^{r}\right)\|f\|_{p}\right. & \left.+\omega_{r, p}(f, t)\right\} \leqslant K_{r, p}\left(f, t^{r}\right) \\
& \leqslant C\left\{\min \left(1, t^{r}\right)\|f\|_{p}+\omega_{r, p}(f, t)\right\}
\end{aligned}
$$

with $C>0$ depending only on $r$. For the spaces $L_{p}\left(I^{m}\right)$ this was given recently by $\mathbf{H}$. Johnen and $\mathrm{K}$. Scherer [7]. We also use some of the ideas from [7] in this section.

We want to examine which of the spaces $B_{p}^{\theta, q}$ are embedded in $W_{\infty}^{2}$ since this will allow us to use Theorem 1 to estimate $\|f-L f\|_{p}$ when $f \in B_{p}^{\theta, q}$.

LeMma 1. (a) For $p=1$, we have $\|f\|_{\infty} \leqslant C\|f\|_{m, 1}$, for each $f \in W_{1}^{m}$. (b) For $1<p<\infty$, we have $\|f\| \leqslant C\|f\|^{m / p, 1}$ for each $f \in B^{m / p, 1}$. 
Proof. Part (a) of this lemma can be found e.g. in [1, p. 100]. Part (b) is readily found in the literature for $L_{p}\left(R^{m}\right)$, see [8] or [3, p. 37]. We include a proof only because the case of $I^{m}$ is not so readily found and yet the ideas are still basically straightforward. One way of proving Lemma 1 is to show the existence of an extension for functions from $B_{p}^{\theta, q}\left(I^{m}\right)$ to $B_{p}^{\theta, q}\left(R^{m}\right)$. However, we want to avoid these extension questions.

Let us first consider part (a). If $\eta$ is a corner of the cube $I^{m}$, let $S_{\eta}=\{x$ : $0<x_{i}<\frac{1}{2}$ when $\eta_{i}=0$, and $\frac{1}{2}<x_{i}<1$, when $\left.\eta_{i}=1\right\}$. We will show that for each $\eta$ and almost all $x \in S_{\eta}$

$$
|f(x)| \leqslant C\|f\|_{m, 1} \text {. }
$$

Since $I^{m}=\cup_{\eta} S_{\eta},(3.2)$ gives part (a).

To establish (3.2), let us first consider the case when $f \in C^{m}\left(I^{m}\right)$. Let $\eta^{\prime}=\left(1-\eta_{1}, 1-\eta_{2}, \ldots, 1-\eta_{m}\right)$.

Take $\varphi$ to be a function in $C^{m}\left(I^{m}\right)$ which vanishes identically on the hyperplanes $x_{i}=\eta_{i}^{\prime}, i=1,2, \ldots, m$, and has the value 1 on $S_{\eta}$. Then with $g(x)=f(x) \varphi(x)$, we have

$$
g(x)=\int_{\eta_{1}^{\prime}}^{x_{1}} \cdots \int_{\eta_{m}^{\prime}}^{x_{m}} \frac{\partial^{m} g\left(s_{1}, \ldots, s_{m}\right)}{\partial x_{1} \partial x_{2} \cdots \partial x_{m}} d s_{1} \cdots d s_{m}
$$

where the boundary terms do not appear because $g$ vanishes where $\varphi$ does. Thus, for $x \in S_{\eta}$

$$
|f(x)|=|g(x)| \leqslant\left\|g_{x_{1} \cdots x_{m}}\right\|_{1} \leqslant C\|\varphi\|_{m, \infty}\|f\|_{m, 1}<C^{\prime}\|f\|_{m, 1}
$$

which is (3.2) for smooth functions.

For an arbitrary $f \in W_{1}^{m}$, we can find a sequence of functions $\left(f_{n}\right)$ in $C^{m}\left(I^{m}\right)$ so that $f_{n} \rightarrow f$ in $W_{1}^{m}$ and $f_{n}(x) \rightarrow f(x)$ a.e., which proves (3.2).

For part (b), we will first establish an inequality for the $J$-functional defined for $u \in W_{p}^{m}$ by

$$
J_{m, p}(u, t)=\max \left(\|u\|_{p}, t\|u\|_{m, p}\right), \quad t>0 .
$$

The embedding theorem rests on showing that

$$
\|u\|_{\infty} \leqslant C \varepsilon^{-m / p} J\left(u, \varepsilon^{m}\right), \quad \varepsilon>0, u \in W_{p}^{m},
$$

with $C$ depending only on $p$ and $m$.

To establish (3.3), we assume first that $u \in C^{m}\left(I^{m}\right)$. Let $\eta$ be any corner of $I^{m}$ and define $S_{\eta}$ as before. Fix $\eta$ and we will show the inequality (3.3) on $S_{\eta}$. For each $0<\varepsilon$, let $T_{\varepsilon}=\left\{t: 0 \leqslant(-1)^{\eta_{i}} t_{i}<\varepsilon\right\}$. When $\varepsilon<1 / 4 m$ and $x \in S_{\eta}$ we have $x+k t \in I^{m}, 0 \leqslant k \leqslant m$, so we can define

$$
\Delta_{t}^{m}(u, x)=(-1)^{m} \sum_{k=0}^{m}(-1)^{k}\left(\begin{array}{c}
m \\
k
\end{array}\right) u(x+k t)
$$

and 


$$
u_{1}(x)=\varepsilon^{-m} \int_{T_{e}}\left\{(-1)^{m+1} \Delta_{t}^{m}(u, x)+u(x)\right\} d t .
$$

It is simple to estimate $\left|u_{1}(x)\right|$ for $x \in S_{\eta}$. Namely,

$$
\begin{aligned}
\left|u_{1}(x)\right| & <2^{m} \varepsilon^{-m} \sup _{1<k<m}\left(\int_{T_{e}}|u(x+k t)|^{p} d t\right)^{1 / p}\left(\int_{T_{e}} d t\right)^{1 / p^{\prime}} \\
& <C \varepsilon^{-m / p}\|u\|_{p}
\end{aligned}
$$

with $C$ depending only on $m$.

We also need an estimate for $u_{2}=u-u_{1}$. Setting $\varphi(\sigma)=u(x+\sigma t)$, $\Delta_{t}^{m}(u, x)=\Delta_{1}^{m}(\varphi, 0)$, where for $\varphi$ we are taking the ordinary one variable differences. Using the Peano kernel representation for the $r$ th difference, we have

$$
\Delta_{t}^{m}(u, x)=\int_{0}^{1} \varphi^{(m)}(\sigma) M(\sigma) d \sigma=\int_{0}^{1} \sum_{|\alpha|=m} t^{\alpha} D^{\alpha}(u, x+\sigma t) M(\sigma) d \sigma .
$$

Here $M$ is nonnegative, has a zero of order $m-1$ at $\sigma=0$, and $\int_{0}^{1} M(\sigma) d \sigma=$ 1. In particular, $\int_{0}^{1} \sigma^{-m / p} M(\sigma) d \sigma$ is bounded for $1<p<\infty$.

Hence, by use of Hölder's inequality we obtain for all $x \in S_{\eta}$

$$
\begin{aligned}
& \left|u_{2}(x)\right|=\left|\varepsilon^{-m} \int_{T_{e}}(-1)^{m+1} \Delta_{t}^{m}(u, x) d t\right| \\
& \quad \leqslant \varepsilon^{-m} \int_{0}^{1} M(\sigma) d \sigma \sum\left\{\int_{T_{e}}\left|D^{\alpha}(u, x+\sigma t)\right|^{p} d t\right\}^{1 / p}\left\{\int_{T_{e}}\left|t^{\alpha}\right|^{p^{\prime}} d t\right\}^{1 / p^{\prime}} \\
& \quad \leqslant \varepsilon^{m / p^{\prime}} \int_{0}^{1} \sigma^{-m / p} M(\sigma) d \sigma \sum\left\{\int_{T_{e}}\left|D^{\alpha}(u, x+\sigma t)\right|^{p} \sigma^{m} d t\right\}^{1 / p} \\
& \leqslant C_{m p}\|u\|_{m, p} \varepsilon^{m / p^{\prime}}
\end{aligned}
$$

where $\Sigma$ is taken over all $\alpha$ such that $|\alpha|=m$.

The estimates (3.4) and (3.5) imply (3.3) for smooth functions $f$ in $C^{m}\left(I^{m}\right)$ and for $0<\varepsilon<1 / 4 \mathrm{~m}$. It is not difficult to conclude that (3.3) actually holds for all $\varepsilon>0$ and, by a denseness argument, for all $f \in W_{p}^{m}$.

Now that we have (3.3), it is an easy matter to verify the embedding (b). From (3.1) it follows that for each integer $k>0$, there is a function $u_{k} \in W_{p}^{m}$ so that

$$
\left\{\begin{array}{l}
\left\|f-u_{k}\right\|_{p}<C\left\{2^{-m k}\|f\|_{p}+\omega\left(2^{-k}\right)\right\}, \\
\left\|u_{k}\right\|_{m p}<C\left\{\|f\|_{p}+2^{m k} \omega\left(2^{-k}\right)\right\},
\end{array}\right.
$$

where $\omega(t)=\omega_{m, p}(f, t)$. Writing $f=\sum_{k=0}^{\infty} g_{k}$ with $g_{k}=u_{k+1}-u_{k}, k=0,1$, $2, \ldots$, and $u_{0}=0,(3.6)$ gives that $J_{m p}\left(g_{k}, 2^{-m k}\right)<C_{m}\left\{2^{-m k}\|f\|_{p}+\omega\left(2^{-k}\right)\right\}$ 
and

$$
\begin{aligned}
\|f\|_{\infty} & \leqslant\left\|u_{1}\right\|_{\infty}+\sum_{k=1}^{\infty}\left\|g_{k}\right\|_{\infty} \\
& \leqslant C_{m, p}\left\{2^{m / p} J_{m, p}\left(u_{1}, 2^{-m}\right)+\sum_{k=1}^{\infty} 2^{m k / p} J_{m p}\left(u_{k}, 2^{-m k}\right)\right\} \\
& \leqslant C_{m, p}\left\{2^{-m / p^{\prime}}\|f\|_{p}+\omega\left(2^{-1}\right)+\sum_{k=1}^{\infty} 2^{-m k / p^{\prime}}\|f\|_{p}+\sum_{k=1}^{\infty} 2^{m k / p} \omega\left(2^{-k}\right)\right\}
\end{aligned}
$$

where we used (3.3). Finally, from the usual properties of the moduli of smoothness it follows that

$$
\sum_{k=1}^{\infty} 2^{m k / p} \omega\left(2^{-k}\right)<C \int_{0}^{1} t^{-\theta} \omega(t) \frac{d t}{t}
$$

This completes the proof of the lemma.

The embeddings of Lemma 1 can be combined with Theorem 1 using interpolation theory to give estimates for $L_{p}$-smoothness $1<p<\infty$. We first consider the simpler cases $p=1$ and $p=\infty$.

THEOREM 2. If $L$ is a positive linear operator mapping $C\left(I^{m}\right)$ into itself and $f \in C\left(I^{m}\right)$, then

$$
\|f-L f\|_{\infty} \leqslant C\left\{\lambda_{\infty}\|f\|_{\infty}+\omega_{2, \infty}\left(f, \lambda_{\infty}^{1 / 2}\right)\right\}
$$

with $C$ depending only on $m$ and the norm of $L$.

For $p=1$, let $L$ be a positive linear operator mapping $L_{1}$ into itself. If $f \in L_{1}\left(I^{m}\right)$, then

$$
\|f-L f\|_{1} \leqslant C\left\{\lambda_{1}\|f\|_{1}+\omega_{m+2,1}\left(f, \lambda_{1}^{1 /(m+2)}\right)\right\}
$$

with $C$ depending only on $m$ and the norm of $L$.

Proof. For $p=\infty$ it is easy to conclude from the estimate (2.1) of Theorem 1 that for each $f \in C\left(I^{m}\right)$

$$
\|f-L f\|_{\infty} \leqslant C K_{2, \infty}\left(f, \lambda_{\infty}\right) \leqslant C\left\{\lambda_{\infty}\|f\|_{\infty}+\omega_{2, \infty}\left(f, \lambda_{\infty}^{1 / 2}\right)\right\},
$$

where the last inequality uses (3.1)

When $p=1$, we first note that from part (a) of Lemma 1 and the estimate (2.1) of Theorem 1 we have that for each $g \in W_{1}^{m+2}$

$$
\|g-L g\|_{1} \leqslant C\|g\|_{2, \infty} \lambda_{1} \leqslant\|g\|_{m+2,1} \lambda_{1} \text {. }
$$

Thus, as in (3.9), for any $f \in L_{1}$ we have

$$
\|f-L f\|_{1} \leqslant C_{L} K_{m+2,1}\left(f, \lambda_{1}\right)<C_{L}\left\{\lambda_{1}\|f\|_{1}+\omega_{m+2, \infty}\left(f, \lambda_{i}^{1 /(m+2)}\right)\right\}
$$

with again the last inequality coming from (3.1). This proves the theorem.

This last theorem gives an estimate for the degree of approximation in 
terms of the modulus of smoothness. This was possible since our endpoint results in these cases where the spaces $W_{2}^{\infty}$ and $W_{1}^{m+2}$. For the general case of $p, 1<p<\infty$, our endpoint spaces involve in general fractional $\theta$, i.e. $\theta=2+m / p$, and then the $K$-functional for interpolation between the endpoint spaces $B_{p}^{\theta, 1}$ and $L_{p}$ is no longer equivalent to a modulus of smoothness. For this reason our estimates will be stated for the classical Lipschitz spaces and not the modulus of smoothness. We let

$$
\operatorname{Lip}\left(\alpha, r ; L_{p}\right)=B_{p}^{\alpha, \infty}=\left\{f \in L_{p}: \omega_{r, p}(f, t)=\mathcal{O}\left(t^{\alpha}\right), 0<\alpha<r, t>0\right\} .
$$

THEOREM 3. Let $1<p<\infty$ and $L$ be any positive linear operator mapping $L_{p}$ into itself. If $r=[2+m / p]+1$ and $f \in \operatorname{Lip}\left(\alpha, r ; L_{p}\right)$ with $\gamma<2+m / p$ $=\theta<r$ then

$$
\|f-L f\|_{p} \leqslant C\|f\|_{p}^{\gamma, \infty} \lambda_{p}^{\gamma / \theta}
$$

with $C$ depending only on $m, p$ and the norm of $L$.

Proof. We know that for each $f \in L_{p}\|f-L f\|_{p}<\left(1+\|L\|_{p}\right)\|f\|_{p}$.

Also, if $f \in B_{p}^{\theta, 1}$ then by Lemma $1 f \in W_{\infty}^{2}$ and consequently by Theorem $1,\|f-L f\|_{p} \leqslant C_{m p}\|f\|_{2, \infty} \lambda_{p} \leqslant C_{m p}\|f\|_{p}^{\theta, 1} \lambda_{p}$.

This time we interpolate between the spaces $L_{p}$ and $B_{p}^{\theta, 1}$. Using the notation in [4] Peetre's interpolation theorem [4, p. 180] gives that for $0<\sigma<1, q=\infty$ and $f \in\left(L_{p}, B_{p}^{\theta, 1}\right)_{\sigma, \infty}$

$$
\|f-L f\|_{p} \leqslant C\|f\|\left(L_{p}, B_{p}^{\theta, 1}\right)_{\sigma, \infty} \lambda_{p}^{\sigma}
$$

where $C$ depends on $m, p, \sigma$ and $\|L\|$.

On the other hand, the reiteration theorem $[4$, p. 178] gives that the spaces $B_{p}^{o \theta, q}$ and $\left(L_{p}, B_{p}^{\theta, 1}\right)_{\sigma, q}, 0<\sigma<1,1<q<\infty$, are isomorphic with equivalent norms. Hence (3.10) and (3.11) are equivalent statements, proving the theorem.

4. Estimates for $L_{p}(\Omega)$. The results of $\S \S 2$ and 3 have analogues for more general domains in $R^{m}$. For example Theorem 1 can be proved for any closed and bounded set $\Omega \subset R^{m}$. Now care must be taken in the definition of $W_{\infty}^{2}(\Omega)$. We follow the approach in $\left[9\right.$, p. 170]. Thus $f \in W_{\infty}^{2}(\Omega)$ if there exist functions $f_{j}, j=0,1, \ldots, m$, so that

$$
\begin{aligned}
& f(x)=f_{0}(y)+\sum_{j=1}^{m} f_{j}(y)\left(x_{j}-y_{j}\right)+R_{0}(x, y), \\
& f_{k}(x)=f_{k}(y)+R_{k}(x, y), \quad k=1,2, \ldots, m,
\end{aligned}
$$

while $\left|f_{j}(x)\right| \leqslant M, j=0,1, \ldots, m,\left|R_{0}(x, y)\right| \leqslant M|x-y|^{2}$, and $\left|R_{k}(x, y)\right|<$ $M|x-y|, k=1,2, \ldots, m$, for all $x, y \in \Omega$. The smallest constant $M$ determines the norm of $f$. 
Using this definition for $W_{\infty}^{2}(\Omega)$, we get the following more general version of Theorem 1.

THEOREM $1^{\prime}$. Let $\Omega$ be a closed bounded set in $R^{m}$, and let $L$ be a positive linear operator mapping $X_{p}(\Omega), 1<p<\infty$, into itself. If $f \in W_{\infty}^{2}(\Omega)$, then

$$
\|f-L f\|_{p}<C\|f\|_{2, \infty} \lambda_{p}
$$

where $\lambda_{p}=\max _{0<i<m+1}\left\|\varphi_{i}-L \varphi_{i}\right\|_{p}(\Omega)$.

To avoid a trivial statement, $\Omega$ is supposed to have positive Lebesgue measure for $1 \leqslant p<\infty$.

The embedding results of $\$ 3$ can also be proved for more general domains. Let $\Omega$ be the closure of some region 0 in $R^{m}$ which has the uniform cone property, see [1, p. 66]. It was shown by H. Johnen and K. Scherer [7] that for such a domain $\Omega$ we still have on $L_{p}(\Omega)$ the equivalence of the $K$-functional

$$
K_{r, p}(f, t)=\inf _{g \in W_{\infty}^{r}(\Omega)}\left\{\|f-g\|_{p}(\Omega)+t\|g\|_{r p}(\Omega)\right\}
$$

and the modulus of smoothness

$$
\omega_{r, p}(f, t)=\sup _{|h|<t}\left\|\Delta_{h}^{r}(f, \cdot)\right\|_{p}\left(\Omega_{r h}\right)
$$

with $\Omega_{r h}=\{x:[x, x+r h] \subseteq \Omega\},[x, y]$ the line segment connecting $x$ and $y$. That is, there exists a constant $C>0$ such that for each $f \in L_{p}(\Omega)$

$$
\begin{aligned}
C^{-1}\left\{\min \left(1, t^{r}\right)\|f\|_{p}\right. & \left.+\omega_{r, p}(f, t)\right\}<K_{r, p}\left(f, t^{r}\right) \\
& <C\left\{\min \left(1, t^{r}\right)\|f\|_{p}+\omega_{r, p}(f, t)\right\}
\end{aligned}
$$

with $C$ independent of $f$ and $t$. The Besov spaces $B_{p}^{\theta, q}(\Omega)$ are defined as before except that we use the modulus of smoothness for $f$ on $\Omega$.

Using a partition of unity type argument as in [7] and estimates similar to those given in $\S 3$, it can be shown when $u \in W_{p}^{m}(\Omega), \varepsilon>0$ and $1<p<\infty$

$$
\|f\|_{\infty}(\Omega)<C \varepsilon^{-\theta} \max \left(\|f\|_{p}(\Omega), \varepsilon^{m}\|f\|_{m, p}\right), \quad f \in W_{p}^{m}(\Omega),
$$

with $\theta=m / p$ and $C$ independent of $f$ and $\varepsilon$. Then the same reasoning as in $\$ 3$ gives the embedding

$$
\|f\|_{\infty}<C\|f\|_{p}^{m / p, 1}(\Omega), \quad f \in B_{p}^{m / p, 1}(\Omega),
$$

with $C$ independent of $f$. For $p=1$, this leads to

$$
\|f\|_{\infty}(\Omega)<C\|f\|_{m, 1}(\Omega), \quad f \in W_{1}^{m}(\Omega),
$$

with $C$ independent of $f$.

These two embeddings give the following generalizations of Theorem 2 and 3.

TheOREM $2^{\prime}$. Let $\Omega=\bar{O}$, where $O$ is a bounded region in $R^{m}$ and $\Omega$ satisfies the cone property $[1, p$. 66]. For $p=\infty$, if $L$ is a positive linear operator 
mapping $C(\Omega)$ into itself, then for all $f \in C(\Omega)$

$$
\|f-L f\|_{\infty} \leqslant C\left\{\lambda_{\infty}\|f\|_{\infty}+\omega_{2, \infty}\left(f, \lambda_{\infty}^{1 / 2}\right)\right\}
$$

with $C$ depending only on $m, \Omega$ and the norm of $L$.

For $p=1$, let $L$ be a positive linear operator mapping $L_{1}(\Omega)$ into itself. If $f \in L_{1}(\Omega)$ then for $f \in L_{1}(\Omega)$

$$
\|f-L f\|_{1} \leqslant C\left\{\lambda_{1}\|f\|_{1}+\omega_{m+2,1}\left(f, \lambda_{1}^{1 /(m+2)}\right)\right\}
$$

with $C$ depending only on $m, \Omega$ and the norm of $L$.

THEOREM 3'. Let $\Omega=\bar{O}$ where $O$ is a bounded region in $R^{m}$ and $\Omega$ has the cone property. Suppose $1<p<\infty$ and $L$ is a positive linear operator mapping $L_{p}(\Omega)$ into itself. If $r=[2+m / p]+1,0<\gamma<2+m / p=\theta<r$ and $f \in$ $\operatorname{Lip}\left(\gamma, r ; L_{p}\right)$ then

$$
\|f-L f\|_{p}(\Omega)<C\|f\|_{p}^{\gamma, \infty} \lambda_{p}^{\gamma / \theta}
$$

with $C$ depending only on $m, p, \Omega$ and the norm of $L$.

ACKNOWLEDGEMENT. The authors would like to thank the referee for pointing out an error.

\section{REFERENCES}

1. R. A. Adams, Sobolev spaces, Academic Press, New York, 1975.

2. H. Berens and R. DeVore, Quantitative Korovkin theorems for $L_{p}$-spaces (Proc. Sympos. on Approximation Theory, Univ. of Texas at Austin, 1976), Academic Press, New York, 1976.

3. Ph. Brenner, V. Thomée and L. B. Wahlbin, Besov spaces and applications to difference methods for initial value problems, Lecture Notes in Math., vol. 434, Springer-Verlag, Berlin and New York, 1975.

4. P. L. Butzer and H. Berens, Semi-groups of operators and approximation, Grundlehren der Math. Wissenschaften, Bd. 145, Springer-Verlag, Berlin, 1967.

5. R. DeVore, Degree of approximation, (Proc. Sympos. on Approximation Theory, Univ. of Texas at Austin, 1976), Academic Press, New York, 1976.

6. G. Freud, On approximation by positive linear methods. I, II, Studia Sci. Math. Hungar. 2 (1967), 62-63; ibid 3 (1968), 365-370.

7. H. Johnen and $\mathrm{K}$. Scherer, On the equivalence of the $K$-functional and moduli of continuity and some applications, Proceedings "Mehrdimensionale konstruktive Funktionentheorie", Oberwolfach, 1976.

8. J. Peetre, Espaces d'interpolation et théorème de Soboleff, Ann. Inst. Fourier (Grenoble) 16 (1966), 279-317.

9. E. M. Stein, Singular integrals and differentiability properties of function, Princeton Univ. Press, Princeton, N.J., 1970.

Mathematischis Institut der Universttät Erlangen-Nurnberg, 852 Erlangen, Federal Republic of Germany (Current address of H. Berens)

Department of Mathematical Sciences, OAKLand University, Rochester, Michigan 48063

Current address (R. De Vore): Department of Mathematical Sciences, University of South Carolina, Columbia, South Carolina 29208 